\title{
Core Facility
}

National Cancer Institute

\section{Source}

National Cancer Institute. Core Facility. NCI Thesaurus. Code C15896.

Shared facilities which provide services of a particular area of expertise. 